\title{
rhAMH inhibits CYP19 and P450scc mRNA expression in granulosa-lutein cells treated with gonadotropin

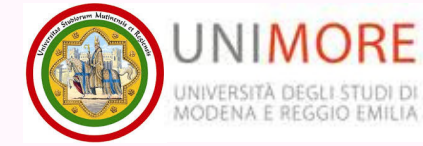

Sandro Sacchi, Federica Marinaro, Daniela Tagliasacchi, Francesca Bastai, Tiziana Marsella, Cindy Argento, Alessandra Tirelli, Simone Giulini, Antonio La Marca

Mother-Infant Department, University of Modena and Reggio Emilia, 41100 Modena, Italy

\section{Introduction}

- Anti-Mullerian hormone (AMH) is a member of transforming growth factor $\beta$ (TGF- $\beta$ )

- Produced by human granulosa cells

- AMH inhibits initiation of primordial follicle growth

- AMH inhibits FSH-stimulated follicle growth

- Negative correlation between AMH retrieved in fluid from small antral follicles and Cyp19A1 mRNA

- $\mathrm{AMH}$ reduces the expression of aromatase CYP19A1 induced by $\mathrm{FSH}$

- Gonadotropins treatment (using LH or FSH) induce strong expression of both aromatases Cyp19A1 and P450scc

\section{Material and Methods}

hGLCs were purified from ovarian follicles of women undergoing in vitro fertilization protocol through a Percoll density gradient then maintained in culture for 6 days to allow the recovery of response to gonadotropins.

The primary hGLCs colture were then incubated for further 24 hours with increasing dosage of rhAMH (range 2-200 ng/ml) to asses the basal transcriptional response of both enzymes. Alternatively, hGLCs were treated for 24 hours with $5 \mathrm{ng} / \mathrm{ml}$ of $\mathrm{rLH}$ or $\mathrm{FSH}$ alone or in combination, and then $\mathrm{AMH}$ at a concentration of $10 \mathrm{ng} / \mathrm{ml}$ was added to colture.

Samples collected from each treatment were processed for RNA extraction followed by retrotranscription to cDNA then evaluated by RT-qPCR using specific pairs of primers. The expression level of both Cyp19A1 and P450scc genes expressed as number of fold changes was normalized by housekeeping gene RPS7. Negative controls were included.

\section{Results}

As shown in Figures 1 - 2 rhAMH was unable to modulate the basal expression of both P450scc and Cyp19A1 in any concentration tested. P450scc (Fig. 3) and Cyp19A1 (Fig. 4) genes were strongly up regulated by rhLH (blue), rhFSH alone (yellow) and by the two goandotropins when combined (green bar). The effect of $20 \mathrm{ng} / \mathrm{ml} \mathrm{rhAMH}$ (gray) added to the colture medium in presence of gonadotropins is also showed in Figs 3 and 4 . AMH completely inhibited the postive effect of gonadotropins on P450scc and Cyp19A1 expression.

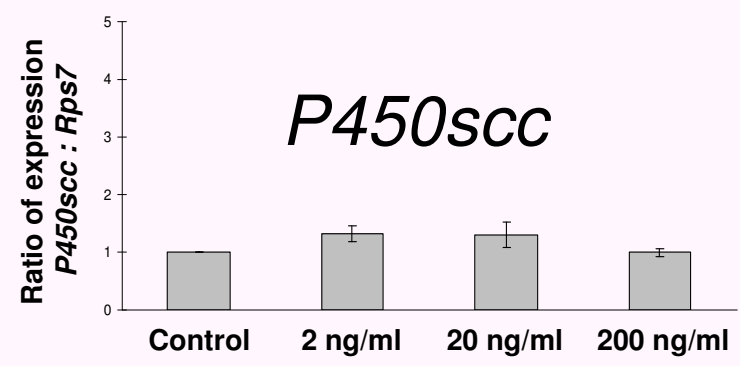

Fig. 1 Effect of increasing concentrations (range $2-200 \mathrm{ng} / \mathrm{ml}$ ) of rhAMH on P450scc expression in hGLCs after 24 hours incubation

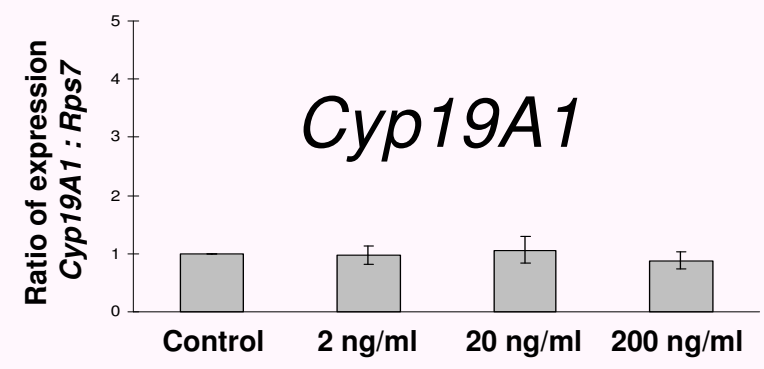

Fig. 2 Effect of increasing concentrations (range $2-200 \mathrm{ng} / \mathrm{ml}$ ) of rhAMH on Cyp19A1 expression in hGLCs after 24 hours incubation

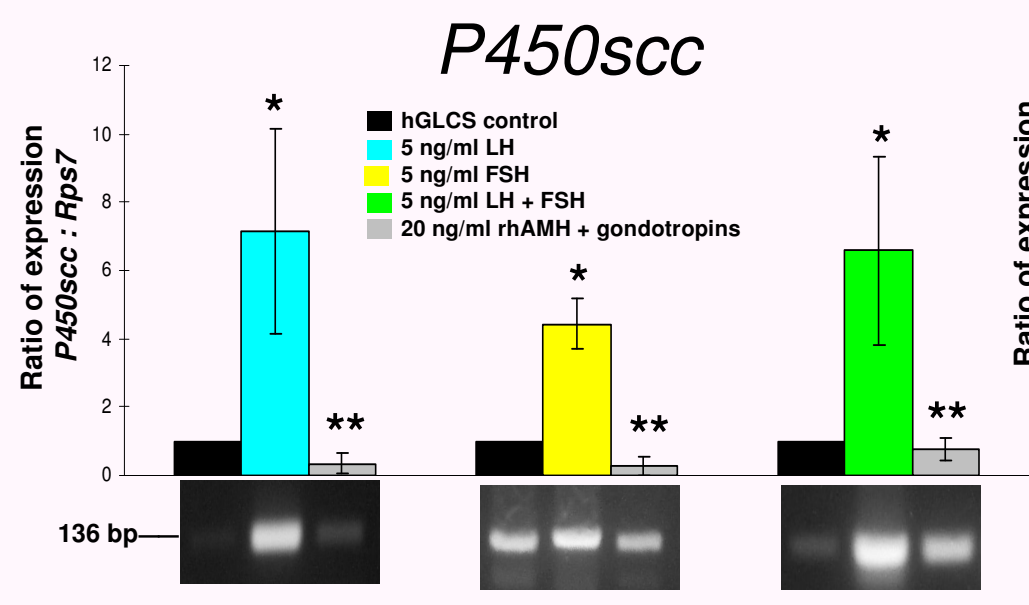

Fig. 3 Effect of gonadotropins and rhAMH alone or combined on P450scc expression in hGLCs after 24 hours incubation.

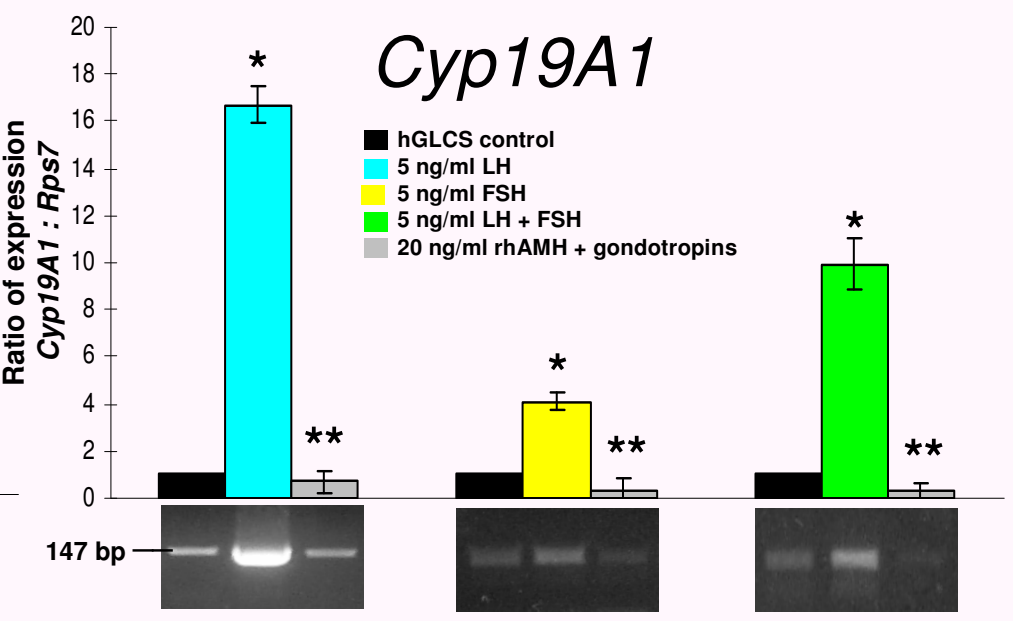

Fig. 4 Effect of gonadotropins and rhAMH alone or combined on Cyp19A1 expression in hGLCs after 24 hours incubation.

\section{Conclusion}

rhAMH reduced the strong transcriptional up regulation of P450scc and Cyp19A1 genes generated by gonadotropins treatment (alone and combined) impairing the enzymes response although rhAMH alone did not affect thier basal expression in any of the concentrations tested. 